\title{
ANÁLISIS DE LAS ACTITUDES HACIA LA ACTIVIDAD MINERA EN LA COMUNIDAD DE COMBAYO, REgión CAJAMARCA
}

\section{ANAlysis of ATTITUdES TOWARDS THE MINING ACTIVITY IN COMBAYO'S COMMUNITY, Cajamarca Region}

\author{
Jorge L. VÁsquez Pacheco \\ Universidad Inca Garcilaso de la Vega \\ Empresa de Servicios Organizacionales, Educativos y Comunitarios Green Planet
}

\begin{abstract}
RESUMEN
Estudio de tipo descriptivo que aplica la metodología transversal mediante una escala original de actitudes, tipo Likert, compuesta inicialmente de 32 reactivos, reducidos a 29 ítems tras el análisis estadístico por medio de la Correlación Item-Test. Dicha escala obtuvo un coeficiente Alfa de Cronbach de ,938, determinando así su consistencia y su validez para el propósito del presente estudio. Los participantes fueron 120 pobladores oriundos de la comunidad de Combayo, región Cajamarca, cuyas edades fluctuaban entre 18 a 60 años (hombres y mujeres), elegidos al azar. No se dio preferencia a uno u otro sexo y todas las dudas fueron resueltas de manera personalizada. Asimismo, tras la aplicación del instrumento se realizaron entrevistas breves a un subgrupo (30) elegido de manera aleatoria con el fin de complementar y contrastar la información obtenida y conocer así si el comunero de Combayo fundamenta la posición que asume. Se obtuvieron cinco factores: calidad de vida, desarrollo comunal, prospección comunal, bienestar psicológico y bienestar físico, cuyos indicadores han permitido establecer los niveles de aprobación respecto a la actividad minera, con los siguientes resultados: para el $80 \%$, la actividad minera es desfavorable; para el 14\%, la actividad minera es favorable; mientras que para el 6\%, la influencia de la actividad minera le resulta neutral. Se concluye que a pesar de la actitud desfavorable hacia la actividad minera en su comunidad, los pobladores la aceptan a nivel conductual, pues mediante las entrevistas se pudo conocer que en general percibían que dicha actividad podía contribuir a su bienestar pero a costa de su calidad de vida (primer factor), siendo mayor la consideración (puntajes de las medias aritméticas) que tienen respecto al desarrollo de su comunidad (segundo factor), la prospección comunal (tercer factor), el bienestar psicológico (cuarto factor) y el bienestar físico (quinto factor). Sin embargo, dicha consideración disminuye respecto a factores contribuyentes a mejorar su calidad de vida. De esta expresión se puede notar que los comuneros de Combayo tienen mayor interés por la obtención de resultados a corto plazo y de satisfacción inmediata respecto a los beneficios que esperan obtener del desarrollo de actividades mineras en su comunidad, lo cual evidencia la presencia de una disonancia cognitiva en dicha comunidad, puesto que estarían reconociendo sus necesidades y también el costo de satisfacer las mismas.
\end{abstract}

Descriptores: actitud, actividad minera, comunidad, disonancia cognitiva 


\begin{abstract}
This is a descriptive study that applies a cross research methodology through the application of an original scale of attitudes, Likert like, composed originally of 32 reagents, reduced to 29 items after the statistical analysis through the Item-Test Correlation. The scale obtained a Cronbach alpha coefficient of, 938 thereby determining the consistency and validity for the purpose of this study. The participants were 120 people natives from the Combayo's community, Region Cajamarca, ranging in age from 18 to 60 years (men and women) selected randomly. No preference was given to any gender and all doubts were resolved in an individual manner. Also, after application of the instrument brief interviews were conducted with a subgroup (30) randomly chosen in order to complement and contrast the information obtained, thus disclosing whether the villager from Combayo has a basis for the position taken. We looked into five factors: Quality of life, community development, community prospecting, psychological wellbeing, and physical wellbeing. Where indicators serve to establish approval levels on mining, with the following results: For $80 \%$ of the subjects mining is unfavorable, for $14 \%$ of the subjects mining is favorable, and for $6 \%$ of the subjects the influence of mining is neutral. We conclude that despite the negative attitude towards mining in this community, residents accept it since they generally perceived that this activity could contribute to their welfare but at the cost of their quality of life (First Factor), but it is of their greater consideration (scores of arithmetic mean) the development of their community (second factor), the communal prospects (third factor), the psychological wellbeing (fourth factor), and physical wellbeing (Fifth Factor). Nevertheless, such consideration falls on contributing to improve their quality of life. One can see that the commoners of Combayo are more interested in obtaining short-term results and immediate satisfaction regarding the benefits expected from the development of mining activities in their community, which shows the presence of cognitive dissonance in that community since they would be recognizing their needs and also the cost of meeting them.
\end{abstract}

Keywords: attitude, mining activity, community, cognitive dissonance

\section{INTRODUCCIÓN}

En la región Cajamarca, la actividad minera cobra auge en el año 1993, cuando la Empresa Minera Yanacocha S.A. toma como sede de sus actividades el valle de la Encañada, colindante a la comunidad de Combayo (zona del estudio, con una altitud aproximada de $3150 \mathrm{msnm}$ ), ubicado a $28 \mathrm{~km}$ de la ciudad de Cajamarca. En dicha comunidad habitan alrededor de 500 familias. La incursión minera generó de modo inevitable cambios en los estilos de vida del poblador originario, específicamente de la comunidad de Combayo, tanto en su medioambiente, en sus estilos de trabajo, como en sus costumbres. Para entenderlo, se debe tomar en cuenta que en la región Cajamarca la población urbana constituye el $28 \%$ y la rural el $72 \%$ [1], ubicándose como la de mayor grado rural del Perú, y que las principales actividades económicas de esta población rural son la agricultura (cereales, menestras, tubérculos, hortalizas, entre otros) [2] y la ganadería (crianza de animales, tanto para la obtención de leche y fabricación de productos derivados, como para la reproducción y venta de carne de vacuno, ovino, caprino, porcino y animales menores) $[3,4]$.

Durante muchos años, se ha planteado que la actividad minera es un fenómeno que genera bienestar y desarrollo, aunque ciertos sectores manifiestan que resulta nociva tanto en el aspecto físico como psicológico y medioambiental [5]. Por tanto, es frente a tal disidencia que el presente estudio pretende servir de puente que ayude a esclarecer tal situación, siguiendo un riguroso manejo de los resultados obtenidos tras la aplicación de la escala original de actitudes, además de un impecable análisis cualitativo de la experiencia adquirida en los días de trabajo en el poblado de Combayo, siempre bajo el criterio imparcial que la formación profesional otorga.

El presente estudio se ejecutó a finales del año 2008, días ricos en interacción con los pobladores 
de Cajamarca y posteriormente con los de la comunidad de Combayo, los cuales lejos de mostrarse huraños, tal y como muchas de las fuentes de referencia lo anunciaban, en especial los medios de comunicación, supieron extender cortesías, compartir sus pensamientos, creencias mágico-religiosas, costumbres, penas y anhelos, además de tatuar en cada una de sus frases el amor a su tierra, a sus montes, a su música, a su aire puro y la tranquilidad que los acompañó a plenitud hace varios años, según refieren. Si analizamos los diversos acontecimientos que llegan a nosotros a través de los medios de comunicación en los últimos 15 años, en los cuales está presente el fenómeno de la actividad minera [6], sería fácil inferir que en Combayo los pobladores se sienten desamparados frente al poder que los funcionarios de la Minera parecen tener, al imperar las gestiones de estos últimos sobre los reiterados y prolongados reclamos de los primeros. Esta situación los ha llevado a tomar la justicia por sus propias manos, a través de tomas de puentes y bloqueos de carreteras, generando una explosión lamentable y ponzońosa de violencia, que lejos de contribuir al desarrollo integral de las comunidades, entorpece toda solución posible [7]. Sin embargo, por una parte, los 15 años que la Minera Yanacocha lleva instalada en Cajamarca han devenido en cierta prosperidad en la ciudad (28\%), con la construcción de hoteles, centros comerciales, locales de diversión, entre otros. Y por otra, ese mismo crecimiento no se siente en las zonas rurales ( $72 \%$ ), ya que en ellas la "mini ganadería" es aún la principal fuente de riqueza, y a su vez, la principal perjudicada por la actividad minera. Tal vez sea por ello que las protestas contra el proceder de la Minera las encabecen los pequeños ganaderos.

En cuanto al impacto ambiental, aunque se aprecia a simple vista en Combayo ( $y$ en general, en las comunidades aledañas a la zona de explotación minera), no existe aún un estudio independiente que lo confirme y sea aceptado de modo bilateral. Solo están las promesas de desarrollo por parte de los funcionarios de la Minera (quienes en la mesa de diálogo aseguran que, según los estudios de su laboratorio de medio ambiente, el circuito cerrado de líquidos que han instalado evita cualquier tipo de contaminación, mediante sus 362 puntos de recojo de muestras de las aguas que salen del área de explotación, con lo cual afirman cumplir con su responsabilidad medioambiental) [8] y la desconfianza de los comuneros, lo cual desemboca en una ruptura del diálogo [9] y por ende en el incremento de daños en la comunidad como conjunto y en cada uno de los pobladores desde su individualidad.

Por tanto, se quiere conocer cuál es la valoración que recibe la actividad minera en los pobladores de la comunidad de Combayo. Para conocer las implicancias de dichas percepciones, el instrumento toma en cuenta cinco factores: calidad de vida (primer factor); desarrollo comunal (segundo factor); prospección comunal (tercer factor); bienestar psicológico (cuarto factor); y bienestar físico (quinto factor). A partir de ellos es posible abstraer la significación de la actividad minera para el poblador desde su individualidad (aspecto cualitativo del estudio), y para la comunidad como ente social (aspecto cuantitativo del estudio).

\section{METODOLOGÍA}

\section{Participantes}

La muestra del estudio estuvo compuesta por 120 pobladores oriundos de la comunidad de Combayo (hombres y mujeres), cuyas edades fluctuaron entre 18 y 60 años.

\section{Instrumento}

Se utilizó una escala original de actitudes hacia la actividad minera, de tipo Likert, compuesta inicialmente de 32 reactivos, que tras el análisis estadístico por medio de la Correlación Ítem-Test quedó reducido a 29 ítems, la misma que tuvo un coeficiente Alfa de Cronbach de ,938.

La escala comprende cinco factores, cada uno de ellos con sus respectivos indicadores; estos son:

- Calidad de vida con cuatro ítems $(1 ; 2 ; 4 ; 5)$

- Desarrollo comunal con 10 ítems (6; 7; 9; 20; $14 ; 16 ; 25 ; 27 ; 28 ; 29)$

- Prospección comunal con cinco ítems (18;21; 23; 24; 26)

- Bienestar psicológico con tres ítems $(8 ; 15 ; 22)$

- Bienestar físico con cuatro ítems $(11 ; 12 ; 13$; 19) 


\section{Procedimiento}

En la comunidad de Combayo, los participantes fueron elegidos al azar, tomándose como único requisito el deseo de colaborar con el desarrollo del cuestionario. No se dio preferencia a uno u otro sexo y todas las dudas fueron resueltas de manera personalizada. Asimismo, tras la aplicación del instrumento se realizaron entrevistas breves a algunos de los participantes (subgrupo de 30) elegidos de manera aleatoria, con el fin de complementar y contrastar la información obtenida mediante el instrumento y conocer si el comunero fundamenta la posición que asume.

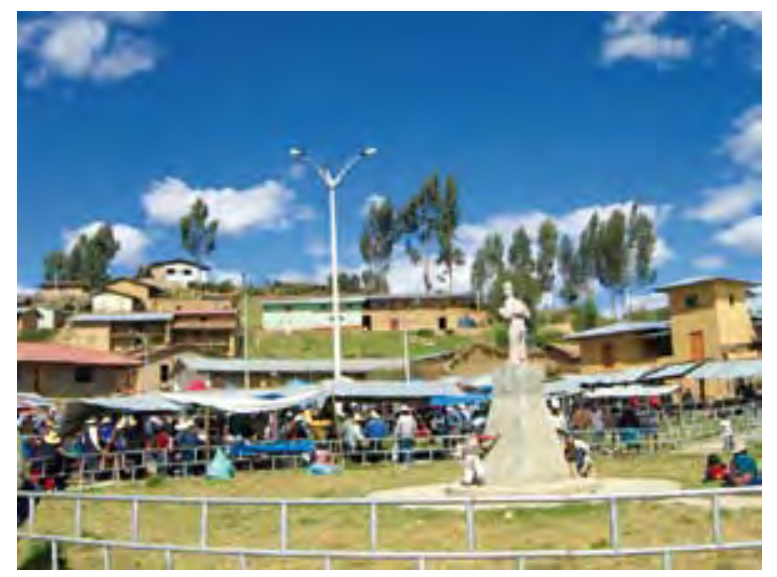

Figura 1: Comunidad de Combayo.

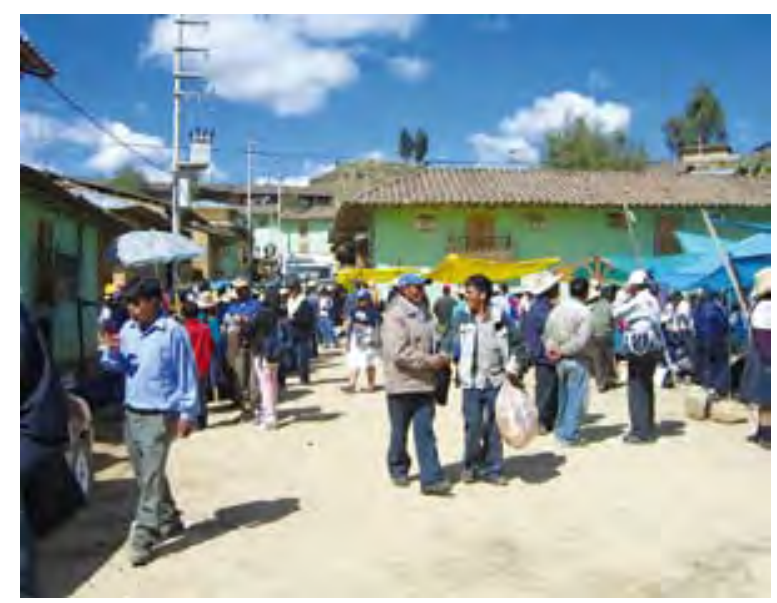

Figura 2: Pobladores de la comunidad de Combayo.

\section{RESULTADOS Y DISCUSIÓN}

\section{Aspecto cuantitativo}

Tras la aplicación de la escala se obtuvo lo siguiente: el $80 \%$ de la muestra (correspondiente a 96 individuos) presenta una actitud desfavorable hacia la actividad minera. El 14\% de la muestra (correspondiente a 17 individuos) tiene una actitud favorable hacia la actividad minera. Mientras que al $6 \%$ de la muestra (correspondiente a siete individuos) la influencia de la actividad minera le resulta neutral.

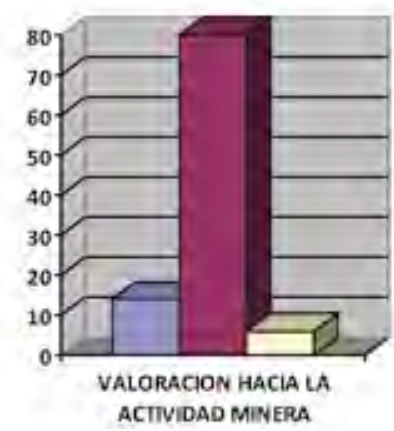

\begin{tabular}{|l|}
\hline E Favorable \\
Desfavorable \\
a Neutral \\
\hline
\end{tabular}

Figura 3: Significancia de la influencia de la actividad minera en los pobladores de la comunidad de Combayo, mostrada en porcentajes.

\section{Aspecto cualitativo}

Tras las entrevistas realizadas, se obtuvo lo siguiente: A pesar de su actitud desfavorable hacia la actividad minera en su comunidad la aceptarían a nivel conductual, pues en general perciben que dicha actividad podría contribuir a su bienestar pero a costa de su calidad de vida (primer factor).

En función a la vertiente cualitativa del estudio, citamos al azar, de manera textual, 10 de las 30 entrevistas realizadas, las cuales expresan de manera compacta las valoraciones generales de esta población:

Lospaisajes quevicuandoera niño están desapareciendo. Yo entiendo que asi debe de ser para que haya progreso, pero no veo tampoco ese progreso que los representantes (funcionarios de la minera) dicen; dónde estará pues, como será.

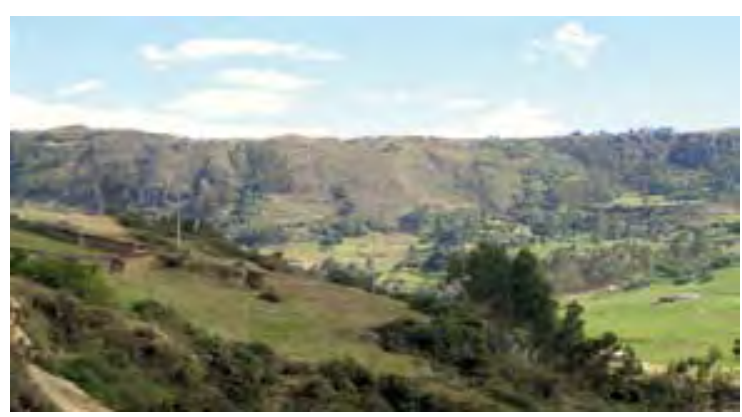

Figura 4: Zonas de hierba seca durante la mejor temporada del año. Fenómeno que se muestra con mayor constancia en los últimos años en esta localidad. 
Poco a poco ha ido cambiando la hierba, mis animalitos no se alimentan igual, ya no engordan, se enferman cuando toman el agua de lluvia que llega oscura.

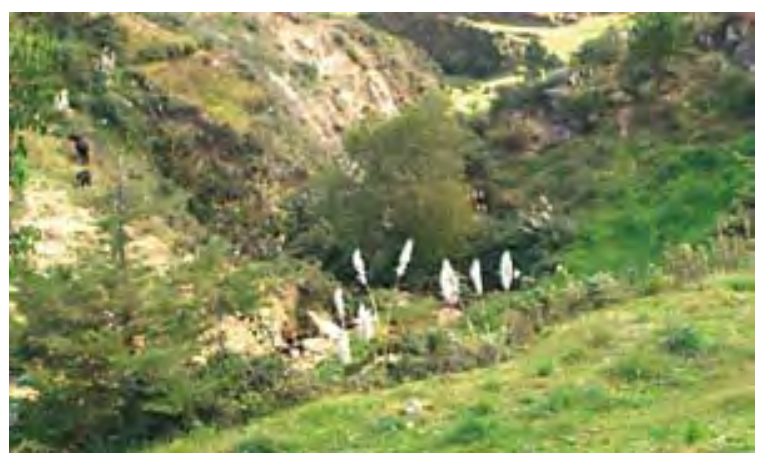

Figura 5: Al lado izquierdo, dos becerros alimentándose con hierba seca, escena cada vez más común en los alrededores del poblado.

Yo estaba con ira, porque de a poco todo lo bonito que ha estado aqui desde que era niño se moría, se desaparecía, como si se lo estuvieran chupando desde arriba. Yo renegaba, quería que se vayan, pero ya no porque nos han dicho que si se van todos vamos a ser pobres, no vamos a tener nada, se van a olvidar de nosotros.

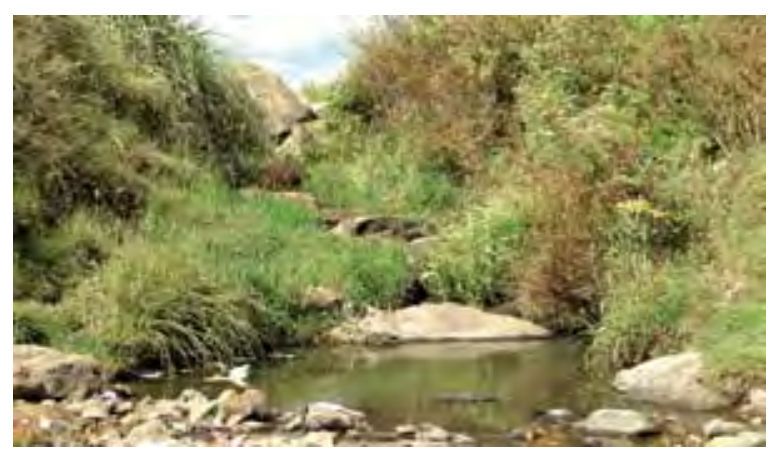

Figura 6: Río típico de la comunidad, antaño cristalino y lleno de peces.

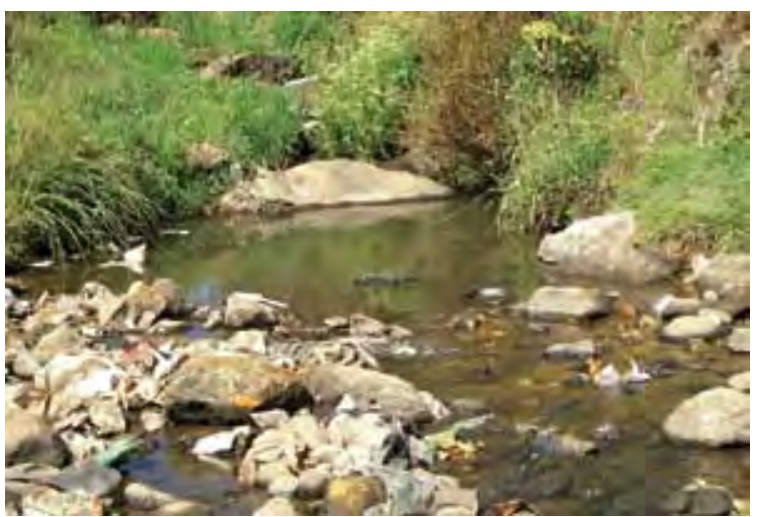

Figura 7: Acercamiento al río antaño cristalino, hoy en vías de desaparición.

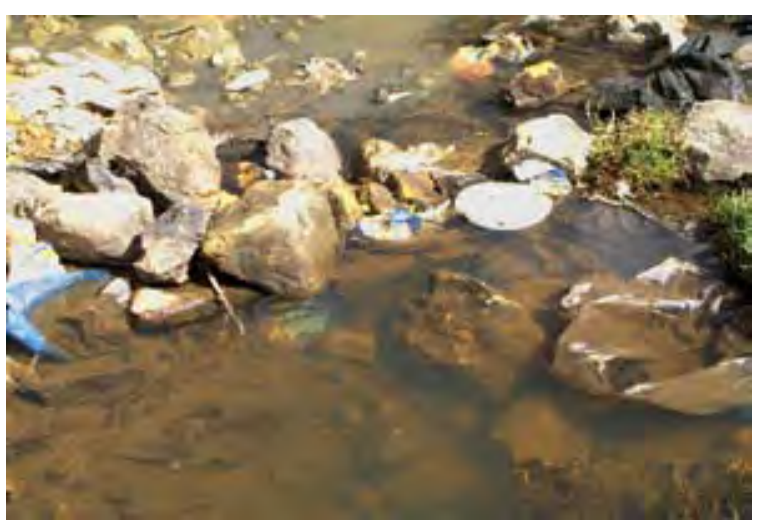

Figura 8: Impacto ambiental en las fuentes hidricas.

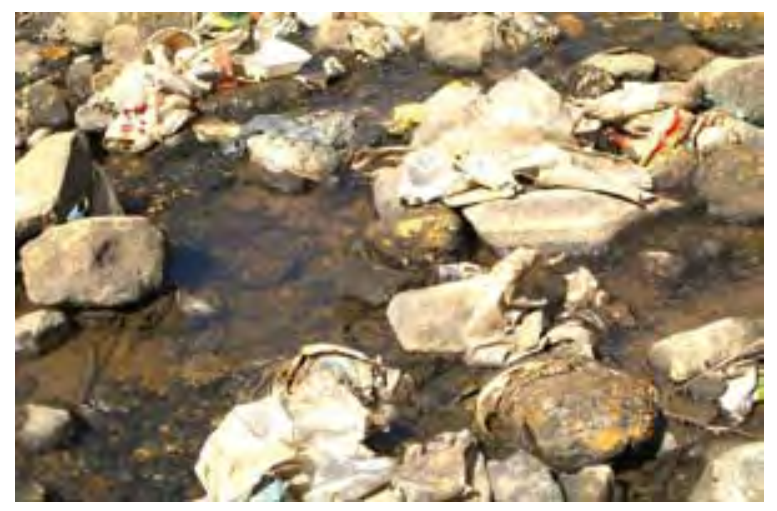

Figura 9: Primer plano del contenido del río que fuera principal fuente de suministro tanto de agua como de peces para uso de la comunidad. Lleno de envases de polietileno, plásticos con logos de productos importados. Totalmente inusuales en el uso de los pobladores oriundos.

Hay nuevas costumbres que enseñan los representantes y como ellos saben mucho, nosotros que no sabemos mucho tenemos que hacer caso nomás, porque si ellos se van todos vamos a ser más pobres.

El agua que antes corrian por aqui, de los rios y las lagunitas se ha ido secando, se ha absorbido.

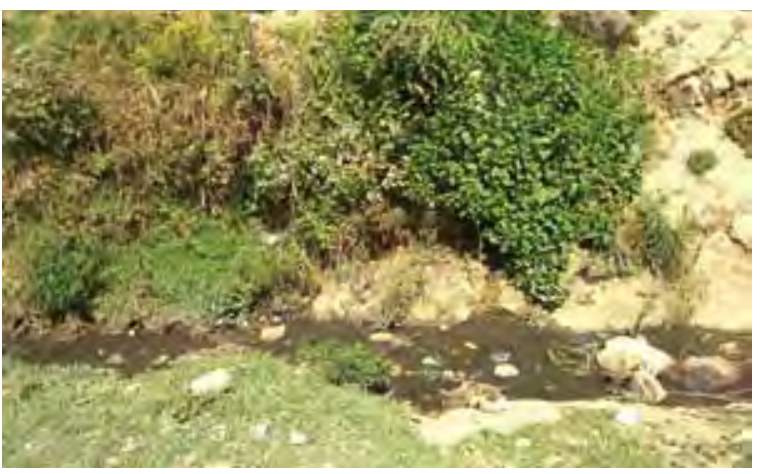

Figura 10: Uno de los rios del cual la comunidad extraía agua para la preparación de alimentos y peces para el consumo, trueque y venta hasta antes de los redireccionamientos de caudales hechos por la Minera. 
Su tecnología trajo que los pescaditos se durmieran, ahora sembrio de papa ya no agarra bien. Ahora han venido rateros, prostitución aqui.

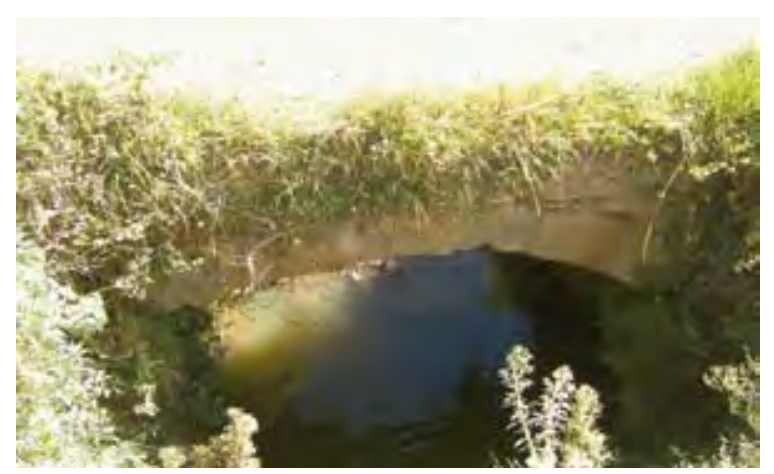

Figura 11: Antiguo puente de piedra bajo el cual se encontraba peces en abundancia, hoy inexistentes.

Nuestras costumbres se han ido olvidando por los jóvenes, parece que más valen las que les gustan a los que bajan con plata al pueblo.

El ruido que hacen molesta, ya no se puede vivir tranquilo, pero dice el gobierno [autoridades de Cajamarca] que asi vamos a progresar y que nuestros familiares van a poder estudiar.

Los jóvenes ya no quieren usar nuestra ropa, se avergüenzan; ahora creen que ser como los que bajan es ser más mejor, que es más bonito.

Antes cuando estábamos en familia, juntos, aunque no tuviéramos cosas bonitas podíamos reírnos, pasear, cantar, bailar, llevar a pastear nuestros animalitos; eso nos llenaba de vida, nos ponía contentos, pero ahora ya no se puede porque asi no se vive, dicen ellos, los que bajan al pueblo.

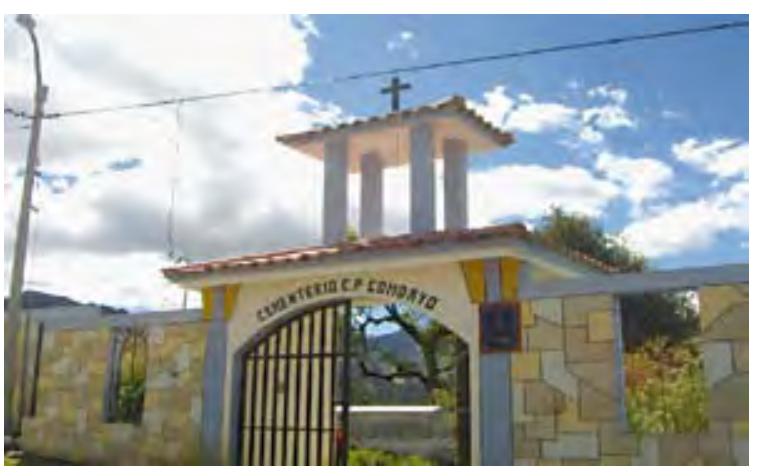

Figura 12: Portal del futuro cementerio de la comunidad, donado por la empresa minera en el 2008.

Podríamos decir que a pesar de la actitud desfavorable hacia la actividad minera en su comunidad, la aceptan a nivel conductual, pues mediante las entrevistas se pudo conocer que los participantes en general percibían que la actividad minera podía contribuir a su bienestar, pero a costa de su calidad de vida (primer factor, cuya satisfacción de necesidades relacionadas tiene como principal característica el mediano o largo plazo). Fue mayor la consideración (puntajes de las medias aritméticas) que tienen respecto al desarrollo de su comunidad (segundo factor); la prospección comunal (tercer factor); el bienestar psicológico (cuarto factor); y bienestar físico (quinto factor). Vale notar que estos últimos cuatro factores se caracterizan porque la satisfacción de necesidades relacionadas puede darse a un corto plazo; sin embargo, esta consideración disminuye respecto a factores contribuyentes a mejorar su calidad de vida.

De esta expresión se puede notar que los comuneros de Combayo muestran mayor interés por la obtención de resultados a corto plazo y de satisfacción inmediata respecto a los beneficios que esperan obtener del desarrollo de actividades mineras en su comunidad $[10,11]$.

Por lo antes expuesto, inferimos que los cambios en el estilo de vida global del comunero consecuencia de la incursión minera es el motivo por el cual demandan la satisfacción inmediata de sus necesidades al momento del estudio. Dicha coyuntura denota la presencia del fenómeno conocido como disonancia cognitiva [12] en los pobladores de la comunidad, lo cual generaría una situación de riesgo latente, pues se conoce que dicho fenómeno es generador de tensión, y dicha tensión, al no resolverse, posee carácter cíclico, siendo la forma en que los pobladores han aprendido a regularla. Son ejemplos las diversas manifestaciones y brotes colectivos de violencia plasmados a lo largo de los años en tomas de puentes, bloqueos de carreteras y desmanes frente a instituciones públicas, los cuales lejos de resolver entorpecen el logro de todo bienestar anhelado.

\section{CONCLUSIONES}

El 80\% dela muestra presenta una actitud desfavorable hacia la actividad minera. El 14\% de la muestra tiene una actitud favorable hacia la actividad minera. Mientras que el 6\% de la muestra la influencia de la actividad minera le resulta neutral. 
A pesar de la actitud desfavorable hacia la actividad minera en su comunidad, la aceptarían a nivel conductual, pues en general perciben que dicha actividad podría contribuir a su bienestar, pero a costa de su calidad de vida (primer factor).

No existe un estudio independiente que confirme el verdadero impacto ambiental en las comunidades aledañas al centro de explotación minera, lo cual trunca la posibilidad de un acuerdo bilateral respecto al presente tema. Así deviene en una ruptura del diálogo en las mesas de negociación, persistiendo el daño en la comunidad como ente social y en cada poblador desde su individualidad.

La información obtenida evidencia que en los pobladores de la comunidad de Combayo se encuentra presente la disonancia cognitiva, teoría planteada por León Festinger [12].

Con el transcurrir de los últimos 15 años, los pobladores han aprendido a regular la tensión provocada por la disonancia cognitiva en diversas manifestaciones y brotes colectivos de violencia, tales como tomas de puentes, bloqueos de carreteras y desmanes frente a instituciones públicas.

Los pobladores fundamentan la valoración desfavorable otorgada a la actividad minera aledaña a su comunidad.

El daño ambiental es real y apreciable, así como nulas las medidas de intervención tomadas por las autoridades competentes.

\section{AGRADECIMIENTOS}

A mi familia, pues sin su apoyo íntegro la presente investigación no hubiera "visto la luz".
Mi agradecimiento especial al Dr. Mateo Mario Salazar Ávalos, maestro y amigo, por la invalorable y desinteresada cooperación hacia la optimización del presente estudio.

\section{REFERENCIAS}

[1] INEI, Censo Nacional de Población y Vivienda 2007.

[2] V. Torres, La agricultura peruana en los tiempos del TLC, Confederación Campesina del Perú, 2005.

[3] Centro Peruano de Estudios Sociales, CEPES, Tipo de ganadería en el Perú, 2006.

[4] Ministerio de Agricultura, Comisión Técnica Plan Ganadero Nacional, Plan Nacional para el Desarrollo Ganadero 2006-2015, Perú, 2006.

[5] Minería, Impactos sociales y ambientales, Movimiento Mundial por los Bosques Tropicales, Secretariado Internacional, Montevideo, Uruguay, ISBN: 9974-7782-5-5, 2004.

[6] Macroconsult, ¿Minería divino tesoro? Retos y perspectivas de la actividad minera peruana, REM Sectorial, Lima, Perú 2005.

[7] Comisión Andina de Juristas, Manual para la construcción de la paz. Negociación en escenarios de conflicto social, 2005.

[8] www.yanacocha.com.pe

[9] R. Vilchez, Avances en la implementación de la agenda social regional de Cajamarca 2007-2011, 2006.

[10] Agenda Social Provincial de Cajamarca 2007.

[11] Agenda Social Regional de Cajamarca 2007.

[12] L. Festinger, A theory of cognitive dissonance, Stanford University Press, Stanford, CA., ISBN 978-0-8047-0911-8, 1957.

E-mail: jorgevasquezpacheco@gmail.com greenplanetperu@hotmail.com 